\title{
Papel do Sistema Endotelina na Nefropatia Diabética
}

\begin{abstract}
RESUMO
A nefropatia diabética (ND) é uma importante complicação crônica do diabetes melito (DM), sendo uma das principais causas dos novos casos de diálise e está associada ao aumento da mortalidade. Os principais fatores de risco são a hiperglicemia, a hipertensão arterial sistêmica (HAS), a dislipidemia e a susceptibilidade genética. O sistema renina-angiotensina (SRA) tem papel importante na gênese e na progressão da ND e existem evidências de interação entre este sistema e as endotelinas. As endotelinas são peptídeos com potente ação vasoconstritora que atuam modulando o tono vasomotor, a proliferação celular e a produção hormonal. Estes peptídeos agem por meio de dois receptores (ET-A e ET-B), que são expressos nas células endoteliais e no músculo liso vascular. A ativação destes receptores nas células renais leva à complexa cascata de alterações, resultando proliferação e hipertrofia das células mesangiais, vasoconstrição das arteríolas aferentes e eferentes e acúmulo de matriz extracelular. Essas alterações hemodinâmicas renais estão associadas com o aparecimento e a progressão da doença renal no DM. Níveis plasmáticos elevados de endotelina-1 (ET-1) têm sido relatados em pacientes com DM e há algumas evidências que sugerem que o aumento da produção de ET-1 poderia levar a dano glomerular. O uso de drogas antagonistas do receptor da ET-1 em situações de DM experimental tem mostrado propriedades nefroprotetoras, reforçando a importância deste sistema na ND. (Arq Bras Endocrinol Metab 2008; 52/4:581-588)
\end{abstract}

Descritores: Endotelina; Diabete Melito; Nefropatia Diabética.

\section{ABSTRACT}

\section{Endothelin System Function in Diabetic Nephropathy.}

Diabetic Nephropathy (DN) is a major chronic complication of diabetes mellitus (DM), and one of the main causes of new cases for dialysis, being associated with increasing mortality. The main risk factors for DN are hypertension, hyperglycemia, dyslipidemia, and genetic susceptibility. The renin-angiotensin system (RAS) plays an important role in genesis and progression of DN and there is evidence of an interrelationship between this system and the endothelins. Endothelins are powerful vasoconstrictor peptides and act as modulators of vasomotor tone, cell proliferation, and hormone production. These peptides act through two types of receptors (ET-A and ET-B) and are expressed on endothelial cells and vascular smooth-muscle cells. Activation of this receptor in renal cells leads to a complex signaling cascade resultanting in stimulation of mesangial cell hypertrophy, proliferation, contraction, and extracellular matrix accumulation. These hemodinamic renal alterations are associated with the onset and progress of renal disease in DM. Elevated endothelin-1 (ET-1) levels have been reported in patients with DM. There is evidence suggesting that an increase in the production of ET-1 leads to glomerular damage. The use of ET receptor antagonists has been reported as renoprotective, correcting the early hemodynamic abnormalities in experimental DM, reinforcing the importance of this system in DN. (Arq Bras Endocrinol Metab 2008; 52/4:581-588)

Keywords: Endothelin-1; Diabetes Mellitus; Diabetic Nephropathy. revisão

\author{
Claudete Maria Zanatta \\ Luís HenRIQue Canani \\ Sandra Pinho Silveiro \\ LUCAS BURTTET \\ Gustavo Nabinger \\ Jorge LUIz GRosS
}

Programa de Pós-Graduação em Ciências Médicas, Endocrinologia da Universidade Federal do Rio Grande do Sul (UFRGS) e Serviço de Endocrinologia do Hospital de Clínicas de Porto Alegre, RS, Brasil.

Recebido em 25/07/2007 Aceito em 22/02/2008 


\section{INTRODUÇÃO}

A NEFropatia diabética (ND) é uma importante complicação do diabetes melito (DM), sendo responsável por aproximadamente um quarto dos casos iniciando diálise na região metropolitana de Porto Alegre, RS (1). Outros estudos relatam incidência ainda maior nos pacientes em início de diálise, chegando próximo a $50 \%$ (2). A ND acomete cerca de $30 \%$ dos pacientes com DM tipo 1, sendo a principal causa de morte nesse grupo e, em pacientes com DM tipo 2 sua prevalência varia de $20 \%$ a $50 \%$, dependendo da origem étnica $(3,4)$. Os pacientes com ND apresentam maior mortalidade desde suas fases iniciais. Além disso, a sobrevida dos pacientes com DM em programas de hemodiálise é menor do que a sobrevida dos pacientes sem DM $(1,5)$.

As alterações estruturais renais relacionadas à ND são caracterizadas por aumento da membrana basal glomerular, espessamento da membrana basal tubular, esclerose mesangial difusa, microaneurismas e arteriosclerose da camada hialina da íntima $(6,7)$. Áreas de intensa expansão mesangial, chamadas nódulos de Kimmelstiel-Wilson ou expansão mesangial nodular, são observadas em torno de $40 \%$ a $50 \%$ dos pacientes que desenvolvem proteinúria, e é uma alteração patognomồnica da ND. Em fases mais avançadas da doença ocorre atrofia tubular e fibrose intersticial $(8,9)$.

Os principais fatores de risco para o desenvolvimento da ND são a hiperglicemia, a hipertensão arterial sistêmica (HAS) (10-12) e a predisposição genética (13-15). Além destes, o sistema renina-angiotensina (SRA) parece ter um papel importante no desenvolvimento e progressão da ND (16-19). No entanto, achados recentes têm demonstrado que outros fatores relacionados ao SRA, como a aldosterona e o sistema endotelina, também têm um papel importante na instalação e na progressão desta complicação $(20,21)$. O objetivo deste trabalho é abordar as evidências de uma possível contribuição do sistema endotelina na gênese da ND.

\section{CARACTERIZAÇÃO DO SISTEMA ENDOTELINA}

As endotelinas são peptídeos vasoconstritores representados pela endotelina-1 (ET-1), endotelina-2 (ET-2) e endotelina-3 (ET-3). Estes peptídeos são produzidos em vários tecidos, onde atuam como moduladores do tônus vascular, proliferação celular e produção hormonal (20-22). Apenas a ET-1 é produzida pelas células endoteliais, sendo, portanto, a que está relacionada à disfunção endotelial. A ET-1 é produzida, em menor proporção, pelas células vasculares do músculo liso, neurônios, células endometriais, células mesangiais dos rins, células de Sertoli e células epiteliais da mama e do pulmão (21). Estímulos como a isquemia, a hipóxia ou a tensão de cisalhamento no vaso induz em minutos à transcrição do RNA mensageiro, à síntese e à secreção da ET-1. Com isso, as células vasculares podem rapidamente ajustar a necessidade de ET-1 para regular o tônus vasomotor. Este peptídeo tem meia-vida plasmática de 4 a 7 minutos e sofre metabolização de primeira passagem $(80 \%$ a $90 \%)$ nos pulmões (21). A ET-1 é considerada um hormônio parácrino, visto que $75 \%$ de sua secreção é liberada no lado sublumial da célula, atuando diretamente no músculo liso vascular (20). Em virtude desta ação parácrina, sua concentração plasmática é muito baixa. Para dosagem da ET-1 sérica é necessária cuidadosa coleta da amostra para evitar degradação do peptídeo in vitro, técnica de extração segura e um método sensível para sua quantificação (22). Apesar de os baixos níveis séricos, vários estudos clínicos correlacionam os níveis da ET-1 sérica com a presença de diversas doenças, como DM tipo 2 e doenças do sistema cardiovascular, como HAS, doença cerebrovascular, insuficiência cardíaca congestiva e infarto agudo do miocárdio (23-27), assim como seu papel como marcador precoce de reoclusão após angioplastia coronariana (28).

A ET-2 é produzida predominantemente nos rins e no intestino e em pequenas quantidades no miocárdio, placenta e útero (2l). É o vasoconstritor mais potente dos três peptídeos. Em animais, ET-2 co-segrega com hipertensão sistólica (29), enquanto no ser humano seu papel não está bem esclarecido. A ET-3 é encontrada em altas concentrações no cérebro e em menor quantidade no trato gastrintestinal, pulmões e rins. Possivelmente regule importantes funções dos neurônios e dos astrócitos (30).

As endotelinas agem nos tecidos-alvo por meio de dois receptores. O receptor do tipo A (ET-A) e o receptor do tipo $\mathrm{B}$ (ET-B), que pertencem à superfamília dos receptores da proteína $\mathrm{G}$ da membrana plasmática $(20,21)$. Os receptores ET-A estão presentes em abundância nas células do músculo liso vascular e nos miócitos cardíacos. Estes apresentam afinidade dez vezes maior para a ET-1 do que para as outras isoformas e é principalmente por meio destes receptores que a ET-1 age, causando vasoconstrição. Os receptores ET-B são expressos predominantemente nas células endoteliais e 
em proporção muito menor nas células do músculo liso vascular $(20,21)$. Estes apresentam a mesma afinidade para as três isoformas da endotelina (21).

\section{FISIOLOGIA DO SISTEMA ENDOTELINA}

O gene da ET-1 está localizado no cromossomo 6 (6p24.1) e contém 5 exons. O gene da ET-2 se localiza no cromossomo 1 ( 1 p34) e contém 5 exons e o gene da ET-3 se localiza no cromossomo 20 (20q13.2 e 20q13.3) e contém 6 exons. $\mathrm{O}$ gene que codifica o receptor ET-A se localiza no cromossomo $4(4 \mathrm{q} 31.22)$ e contém 8 exons, enquanto o gene do ET-B tem duas isoformas e localiza-se no cromossomo 13 (13q22) e tem 7 exons (http://www.ncbi.nlm.nih.gov/GenBank).

A expressão dos receptores da endotelina (ET-A e ET-B) é regulada em paralelo com a expressão da ET-1 (31). Fatores físicos, bioquímicos e humorais regulam a transcrição do gene da ET-1 estimulando a síntese da pré-proendotelina-1, que contém 203 aminoácidos. Este pré-hormônio é então transformado pelas endopeptidases em big-endotelina-1, que é a forma circulante no plasma, mas que é funcionalmente inativa. As enzimas de conversão da endotelina, quimases e metaloproteases clivam a big-endotelina entre as posições 21 (triptofano) e 22 (valina), liberando a forma ativa da ET-1 com 21 aminoácidos (21).

Em situações de hipóxia, ocorre estímulo para produção de ET-1 e do receptor ET-A nas células endoteliais e nas células do músculo liso vascular concomitantemente. A ativação dos receptores da ET-A estimula a hidrólise da fosfolipase $\mathrm{C}$ que leva ao aumento do cálcio intracelular, causando vasoconstrição e ativando a proteína $\mathrm{C}$ quinase que resulta proliferação tecidual $(20,21)$. A vasoconstrição persiste após a ET-1 ser removida do receptor, provavelmente em virtude de a concentração de cálcio intracelular permanecer elevada. A presença de óxido nítrico encurta o período de vasoconstrição por acelerar o retorno do cálcio intracelular ao valor basal (21).

O receptor ET-B está presente em maior quantidade nas células endoteliais e a sua ativação causa liberação de substâncias vasodilatadoras, como o óxido nítrico e a prostaciclina, levando à vasodilatação inicial, seguida por vasoconstrição (31). Este receptor é encontrado em menor proporção nas células do músculo liso vascular, onde sua ativação causa vasoconstrição (Figura 1).

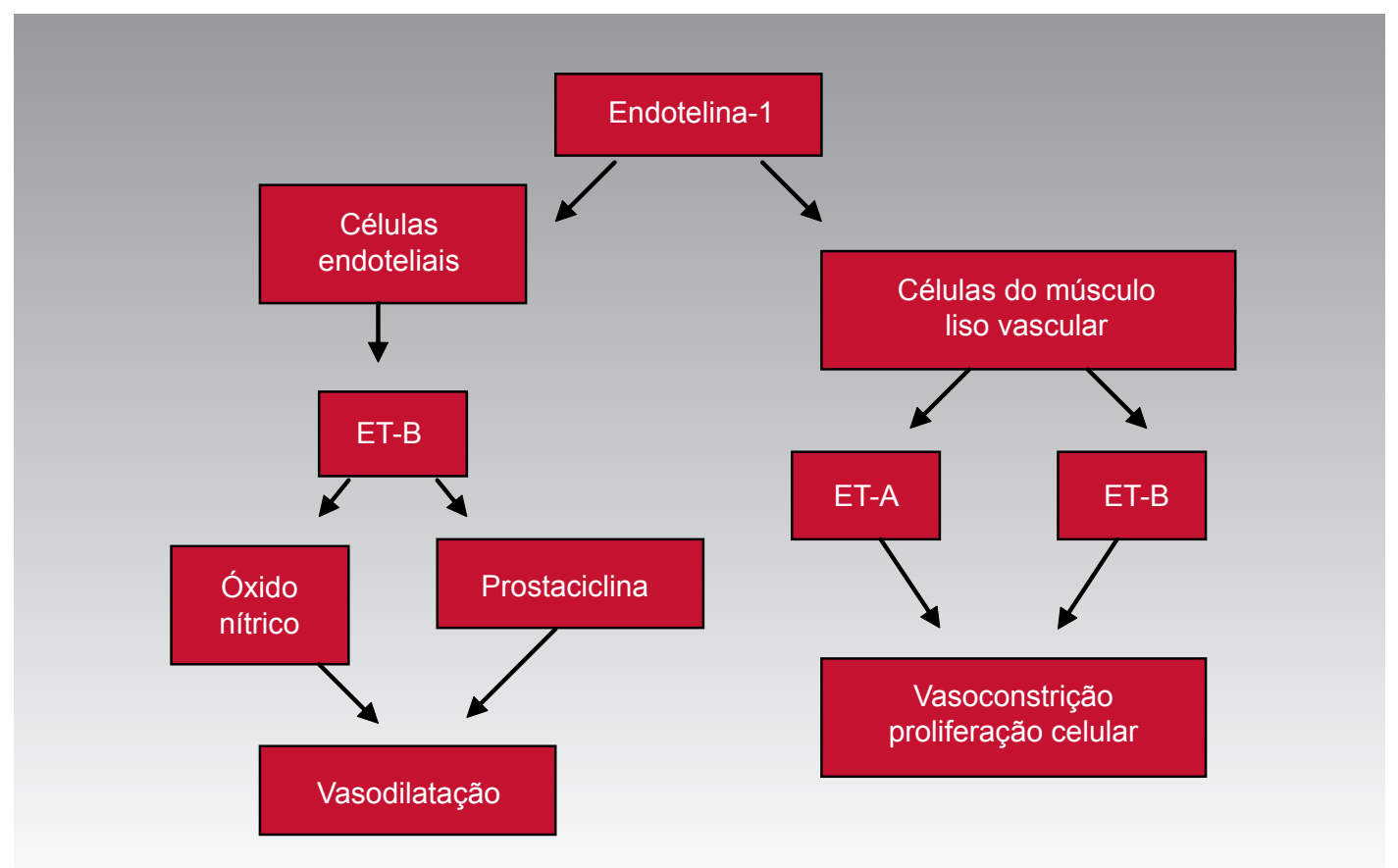

Figura 1. Ação da endotelina pelos receptores ET-B nas células endoteliais causando vasodilatação, e pelos receptores ET-A e ET-B nas células do músculo liso vascular causando vasoconstrição. 


\section{ENDOTELINA-1}

Das três endotelinas, a ET-1 é a principal representante do sistema endotelina relacionada a distúrbios hemodinâmicos e de disfunção endotelial. A ET-1 e o receptor ET-A têm provavelmente importante papel na manutenção do tônus vasomotor basal (32). Esta endotelina tem efeito vasoconstritor cem vezes maior que a norepinefrina, sendo um dos mais potentes vasoconstritores já identificados (21). Este efeito é mediado principalmente pela ativação do receptor da ET-A nas células do músculo liso vascular (33). A ET-1 também potencializa a vasoconstrição causada pela norepinefrina e pela epinefrina, as quais estimulam a produção de ET-1 (34).

A ET-1 interage com outros fatores vasoativos e sua produção é estimulada por vários hormônios, peptídeos vasoativos, fatores de crescimento e condições de aumento da tensão de cisalhamento na parede dos vasos. Substâncias vasodilatadoras, como o óxido nítrico e as prostaciclinas, inibem a sua produção (21) (Tabela 1).

Tabela 1. Fatores relacionados a expressão e síntese da ET-1.

\begin{tabular}{ll}
\hline Estimuladores & Inibidores \\
\hline Angiotensina II & Óxido nítrico \\
\hline Catecolaminas & Prostaglandinas \\
\hline Fator de crescimento & Prostaciclina \\
\hline Hipóxia & Hormônio atrial natriurético \\
\hline Insulina & Endotelina-3 \\
\hline Lipídios (LDL, HDL) & Estrogênios \\
\hline Tensão de cisalhamento & \\
\hline Hiperglicemia & \\
\hline
\end{tabular}

O endotélio dos vasos regula o tônus e o crescimento vascular por intermédio do equilíbrio entre fatores de relaxamento e de contração. As ações mitogênicas e vasoconstritoras da ET-1 são contrabalançadas por fatores vasodilatadores e anticrescimento, como as prostaciclinas e o fator de relaxamento derivado do endotélio (35). Alterações metabólicas que estão presentes no DM, como o hiperinsulinismo, a hiperglicemia e a hiperlipidemia, estimulam diretamente a liberação da ET-1 pelas células endoteliais e a expressão de seus receptores (20). A exposição prolongada das células endoteliais e do músculo liso vascular a alterações metabólicas do DM promove aterosclerose e outras alterações morfológicas nos vasos, que indiretamente afetam a liberação e a ação da
ET-1 em vários órgãos-alvo (20). Vários estudos têm descrito aumento dos níveis séricos de ET-1 em pacientes com DM (23-25) em estados de resistência à insulina $(26,27)$. Além disso, o uso de antagonistas dos receptores da ET-1 melhora a resposta vasodilatadora em pacientes com HAS (36-38). Em modelos de animais diabéticos, estes fármacos apresentam efeito protetor no desenvolvimento e progressão da ND (39-41).

\section{ENDOTELINA-1 E NEFROPATIA DIABÉTICA}

O rim é um importante local de produção e também é alvo da ação da ET- 1 . As células mesangiais são as principais células que produzem e sofrem ação da ET-1 no rim (42), entretanto as células epiteliais glomerulares e tubulares também produzem ET-1 (43). Os receptores ET-A estão presentes nas artérias arqueadas e vasa reta e os receptores ET-B são encontrados predominantemente nos ductos coletores, sugerindo diferentes papéis na reabsorção de sal e água. Ambos receptores são encontrados no glomérulo (31).

Os efeitos hemodinâmicos da ET-1 no rim são aumentar a resistência vascular renal por vasoconstrição das arteríolas aferentes e eferentes e artérias arqueadas e interlobulares. Conseqüentemente, há redução do fluxo sanguíneo, taxa de filtração glomerular e inibição da reabsorção de sal e de água. A ET-1 também causa proliferação celular glomerular e acúmulo de proteínas na matriz extracelular (31). A administração sistêmica de ET-1 reduz a taxa de filtração glomerular e pode causar natriurese, um mecanismo que provavelmente está relacionado ao efeito do receptor ET-A (44).

Como descrito anteriormente, existe interação da ET-1 com outras substâncias vasoativas, como a angiotensina II (Ang II) $(45,46)$. A inibição da produção de Ang II, estimulada pela ET-1 ou a inibição direta da ação da ET-1 pode prevenir efeitos da Ang II de estímulo à hipertrofia cardíaca $(47,48)$. A Ang II, reconhecidamente relacionada à ND, tem sido identificada como um potente estimulador da secreção de ET-l (37) e alguns de seus efeitos são dependentes de maior estímulo da ET-1 (36). Em culturas de células mesangiais renais, a síntese de matriz protéica induzida pela ET-1 pode ser prevenida com o uso de inibidores da enzima conversora da angiotensina e, no tecido vascular, o efeito da Ang II é atenuado pelo uso de antagonistas da ET-1 (38). Estas evidências mostram importante inter-relação dessas duas substâncias vasoconstritoras na lesão renal. 
Recentes estudos têm discutido o papel da ET-1 na fase inicial da ND. Foram encontrados em pacientes com DM tipo 2 níveis plasmáticos elevados de ET-1, independente da presença de doença microvascular, porém os pacientes com DM e microalbuminúria tinham níveis de ET-1 significativamente mais elevados do que normoalbuminúricos e hipertensos sem DM (25). Por outro lado, um estudo prévio com pequeno número de pacientes mediu os níveis séricos de ET-1 em pacientes com DM microalbuminúricos, macroalbuminúricos e controles sem DM e encontrou níveis significativamente mais elevados apenas no grupo com macroalbuminúria (49). Em um número maior de pacientes com DM tipo 2 e função renal preservada, demonstramos que os níveis de ET-1 estão aumentados de maneira crescente nos pacientes micro e macroalbuminúricos, quando comparados aos pacientes normoalbuminúricos (50) (Figura 2).
A atividade da ET-1 endógena no receptor ET-A (51) e a produção renal de ET-1 estão aumentadas em indivíduos com DM (25). Também foi observado que a expressão do gene da ET-1 está aumentada em rins de ratos diabéticos, assim como em culturas de células mesangiais de rato expostas à alta concentração de glicose (52). Estes achados reforçam a hipótese de que a ativação precoce do endotélio vascular levaria ao aumento da produção e expressão de ET-1, e isso favoreceria a instalação da microalbuminúria (25). Estes achados são reforçados por um estudo que avaliou a função endotelial em pacientes com DM tipo 1 por meio da dosagem seriada de fator de von Willebrand (vWF) por período de 64 meses. Neste estudo, foi demonstrado que a disfunção endotelial, estimada pelo nível plasmático do fator de vWF, precedeu o início de microalbuminúria em três $\operatorname{anos}(53)$.

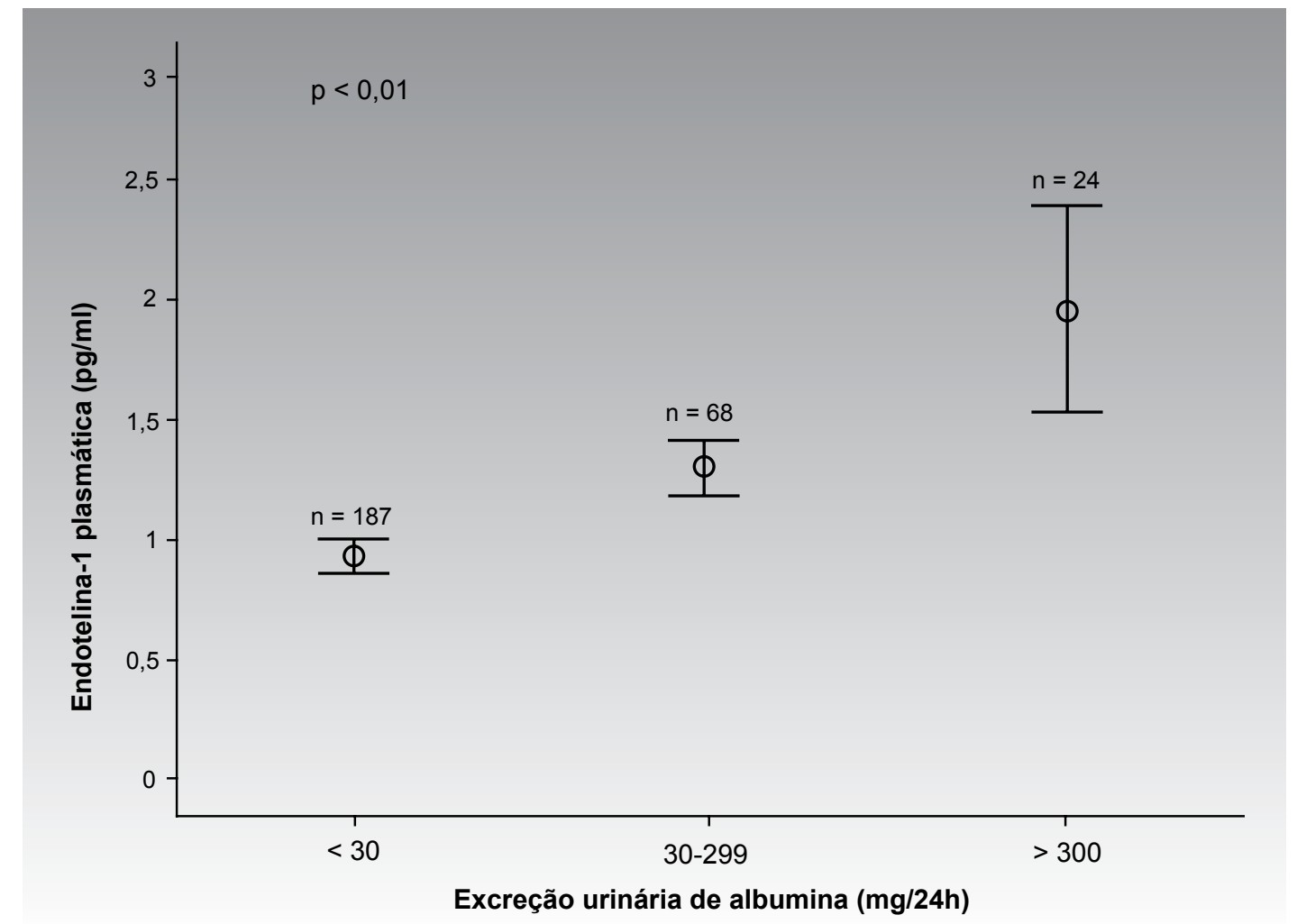

O circulo central é a média com intervalo de confiança de 95\%. P < 0,01 para comparação de todos os grupos entre si.

Figura 2. Níveis plasmáticos de endotelina-1 em pacientes com DM tipo 2 conforme excreção urinária de allbumina. 
O papel das endotelinas na regulação da hemodinâmica renal, assim como no crescimento da matriz extracelular, faz que os genes envolvidos neste sistema sejam candidatos naturais implicados no desenvolvimento da ND. Como citado anteriormente, a hiperglicemia per se leva ao aumento na expressão do gene da ET-1 (54). Entretanto, não existem estudos que avaliem se as alterações funcionais nesses genes poderiam estar associadas à presença de ND. Alguns dos polimorfismos descritos no gene que codifica o receptor A (EX8nt211, EX8ntl363) estão associados à maior pressão de pulso (55). Entretanto, nenhum deles foi analisado em relação à presença de ND.

\section{MEDICAÇÕES QUE ATUAM NO SISTEMA ENDOTELINA}

Estudos em modelos animais demonstram que ocorre normalização da pressão glomerular e diminuição de deposição de proteínas na matriz extracelular, assim como diminuição da excreção urinária de albumina, com o uso do bloqueador do receptor ET-A/ET-B (bosentan) (40). Nestes animais, o uso do bosentan apresentou efeito nefroprotetor, corrigindo tanto a hiperfiltração inicial quanto a evolução para nefropatia clínica (41). Hocher e cols. (56) estudaram o uso de antagonista seletivo do ET-A (LU 135252) em comparação com o antagonista combinado ET-A/ET-B (LU 224332) em ratos, e mostraram que os dois antagonistas normalizaram a expressão da fibronectina e do colágeno tipo IV dentro do glomérulo, que estava aumentada após 36 semanas da indução do DM, e reduziram em $50 \%$ a proteinúria em relação aos controles. $\mathrm{O}$ efeito antifibrótico parece ser mediado via receptor ET-A. Um estudo em pacientes com DM tipo 2 obesos, com resistência à insulina, mostrou melhora da vasodilatação endotélio-dependente com o uso do bloqueador do ET-A (BQ123) (57). Estes resultados podem ter importantes implicações no tratamento e na prevenção da ND, assim como de outras complicações do DM.

\section{CONCLUSÕES E PERSPECTIVAS}

As evidências atuais indicam que a ET-1 seja um importante marcador da disfunção endotelial que leva à hipertrofia vascular, aterogênese e glomeruloesclerose. $\mathrm{O}$ aumento dos níveis séricos tem sido descrito em pa- cientes com DM, especialmente naqueles que apresentam aumento da excreção urinária da albumina. Reforçada por estudos de intervenção, pode se inferir que esta associação tenha efeito causal na etiologia da ND e que não seja meramente secundária à presença desta (epifenômeno). A aplicação clínica de bloqueadores da ação das endotelinas é promissora e, caso se confirme, poderá ser uma importante opção terapêutica no tratamento e na prevenção desta complicação do DM. Por fim, estudos que avaliem se existem variantes gênicas associadas à ND permitiriam identificação precoce de grupos de indivíduos suscetíveis, nos quais a intervenção mais precoce e agressiva seria benéfica.

\section{REFERÊNCIAS}

1. Bruno RM, Gross JL. Prognostic factors in Brazilian diabetic patients starting dialysis: a 3.6-year follow-up study. J Diabetes Complications. 2000;14:266-71.

2. IV. Patient characteristics at the start of ESRD: data from the HCFA medical evidence form. Am J Kidney Dis. 1999;34:S63-73.

3. Scheffel RS, et al. Prevalence of micro and macroangiopatic chronic complications and their risk factors in the care of out patients with type 2 diabetes mellitus. Rev Assoc Med Bras. 2004;50:263-7.

4. Adler Al, et al. Development and progression of nephropathy in type 2 diabetes: the United Kingdom Prospective Diabetes Study (UKPDS 64). Kidney Int. 2003;63:225-32.

5. Nelson RG, Pettitt DJ, Carraher MJ, Baird HR, Knowler WC. Effect of proteinuria on mortality in NIDDM. Diabetes. 1988;37:1499-504.

6. Mauer SM, Steffes MW, Brown DM. The kidney in diabetes. Am J Med. 1981;70:603-12.

7. Kimmestiel P, Wilson C. Intercapillary lesions in the glomeruli of kidney. Am J Pathol. 1936;12:83-97.

8. Fioretto $\mathrm{P}$, et al. Patterns of renal injury in NIDDM patients with microalbuminuria. Diabetologia. 1996;39:1569-76.

9. Mauer SM, et al. Structural-functional relationships in diabetic nephropathy. J Clin Invest. 1984;74:1143-55.

10. Tight blood pressure control and risk of macrovascular and microvascular complications in type 2 diabetes: UKPDS 38. UK Prospective Diabetes Study Group. BMJ. 1998;317:703-13.

11. Hollenberg, NK. Treatment of the patient with diabetes mellitus and risk of nephropathy: what do we know, and what do we need to learn? Arch Intern Med. 2004;164:125-30.

12. Gross JL, et al. Diabetic nephropathy: diagnosis, prevention, and treatment. Diabetes Care. 2005;28:164-76.

13. Krolewski, AS. Genetics of diabetic nephropathy: evidence for major and minor gene effects. Kidney Int. 1999;55:1582-96.

14. Canani LH, Gerchman F, Gross, JL. Familial clustering of diabetic nephropathy in Brazilian type 2 diabetic patients. Diabetes. 1999;48:909-13.

15. Khoury MJ, Beaty TH, Liang KY. Can familial aggregation of disease be explained by familial aggregation of environmental risk factors? Am J Epidemiol. 1988;127:674-83. 
16. Gross ML, Amann K. Progression of renal disease: new insights into risk factors and pathomechanisms. Curr Opin Nephrol Hypertens. 2004;13:307-12.

17. Lasaridis AN, Sarafidis PA. Diabetic nephropathy and antihypertensive treatment: what are the lessons from clinical trials? Am J Hypertens. 2003;16:689-97.

18. Mogensen CE, et al. Prevention of diabetic renal disease with special reference to microalbuminuria. Lancet. 1995;346:1080-4.

19. Penno G, et al. Effect of angiotensin-converting enzyme (ACE) gene polymorphism on progression of renal disease and the influence of ACE inhibition in IDDM patients: findings from the EUCLID Randomized Controlled Trial. EURODIAB Controlled Trial of Lisinopril in IDDM. Diabetes. 1998;47:1507-11.

20. Hopfner RL, Gopalakrishnan V. Endothelin: emerging role in diabetic vascular complications. Diabetologia. 1999;42:1383-94.

21. Levin ER. Endothelins. N Engl J Med. 1995;333:356-63.

22. Abdel-Sayed $S$, et al. Measurement of plasma endothelin-1 in experimental hypertension and in healthy subjects. Am J Hypertens. 2003;16:515-21.

23. Schneider JG, et al. Elevated plasma endothelin-1 levels in diabetes mellitus. Am J Hypertens. 2002;15:967-72.

24. Seligman BG, Biolo A, Polanczyk CA, Gross JL, Clausell N. Increased plasma levels of endothelin 1 and von Willebrand factor in patients with type 2 diabetes and dyslipidemia. Diabetes Care. 2000;23:1395-400.

25. De Mattia G, et al. Role of plasma and urinary endothelin-1 in early diabetic and hypertensive nephropathy. Am J Hypertens. 1998;11:983-8.

26. Ferri $C$, et al. Plasma endothelin-1 levels in obese hypertensive and normotensive men. Diabetes 1995;44:431-6.

27. Ferri $C$, et al. Circulating endothelin-1 levels in obese patients with the metabolic syndrome. Exp Clin Endocrinol Diabetes. 1997;105 Suppl 2:38-40.

28. Kyriakides ZS, et al. Coronary vasoconstriction after coronary angioplasty is attenuated by endothelin a receptor antagonism. Am J Cardiol. 2001;87:1011-3; A5.

29. Ohkubo $S$, et al. Specific expression of human endothelin-2 (ET-2) gene in a renal adenocarcinoma cell line. Molecular cloning of cDNA encoding the precursor of ET-2 and its characterization. FEBS Lett. 1990;274:136-40.

30. Dupin E, Glavieux C, Vaigot P, Le Douarin NM. Endothelin 3 induces the reversion of melanocytes to glia through a neural crest-derived glial-melanocytic progenitor. Proc Natl Acad Sci U S A. 2000;97:7882-7.

31. Simonson MS. Endothelins: multifunctional renal peptides. Physiol Rev. 1993;73:375-411.

32. Haynes WG, Webb DJ. Contribution of endogenous generation of endothelin-1 to basal vascular tone. Lancet. 1994;344:852-4

33. Arai $\mathrm{H}$, Hori $\mathrm{S}$, Aramori I, Ohkubo $\mathrm{H}$, Nakanishi S. Cloning and expression of a cDNA encoding an endothelin receptor. Nature. 1990;348:730-2

34. Tabuchi $Y$, et al. Endothelin inhibits presynaptic adrenergic neurotransmission in rat mesenteric artery. Biochem Biophys Res Commun. 1989;161:803-8.

35. Vane JR, Anggard EE, Botting RM. Regulatory functions of the vascular endothelium. N Engl J Med. 1990;323:27-36.

36. Barton M, Luscher TF. Endothelin antagonists for hypertension and renal disease. Curr Opin Nephrol Hypertens. 1999;8:549-56.
37. Kohno $\mathrm{M}$, et al. Plasma immunoreactive endothelin in essential hypertension. Am J Med. 1990;88:614-8.

38. Cardillo C, Campia U, Kilcoyne CM, Bryant MB, Panza JA. Improved endothelium-dependent vasodilation after blockade of endothelin receptors in patients with essential hypertension. Circulation. 2002;105:452-6.

39. Sugimoto K, Tsuruoka S, Fujimura A. Renal protective effect of YM598, a selective endothelin ET(A) receptor antagonist, against diabetic nephropathy in OLETF rats. Eur J Pharmacol. 2002;450:183-9.

40. Cosenzi A, et al. Nephroprotective effect of bosentan in diabetic rats. J Cardiovasc Pharmacol. 2003;42:752-6.

41. Ding SS, et al. Chronic endothelin receptor blockade prevents both early hyperfiltration and late overt diabetic nephropathy in the rat. J Cardiovasc Pharmacol. 2003;42:48-54.

42. Sorokin A, Kohan DE. Physiology and pathology of endothelin-1 in renal mesangium. Am J Physiol Renal Physiol. 2003;285:F579-89.

43. Herizi A, Jover B, Bouriquet N, Mimran A. Prevention of the cardiovascular and renal effects of angiotensin II by endothelin blockade. Hypertension. 1998;31:10-4.

44. Ferrario RG, Foulkes $R$, Salvati $P$, Patrono C. Hemodynamic and tubular effects of endothelin and thromboxane in the isolated perfused rat kidney. Eur J Pharmacol. 1989;171:127-34.

45. Sadoshima J, Xu Y, Slayter HS, Izumo S. Autocrine release of angiotensin II mediates stretch-induced hypertrophy of cardiac myocytes in vitro. Cell. 1993;75:977-84.

46. Ito $\mathrm{H}$, et al. Endothelin-1 is an autocrine/paracrine factor in the mechanism of angiotensin II-induced hypertrophy in cultured rat cardiomyocytes. J Clin Invest. 1993;92:398-403.

47. Pfeffer MA, et al. Effect of captopril on mortality and morbidity in patients with left ventricular dysfunction after myocardia infarction. Results of the survival and ventricular enlargement trial. The SAVE Investigators. N Engl J Med. 1992;327:669-77.

48. Moreau $P$, et al. Angiotensin II increases tissue endothelin and induces vascular hypertrophy: reversal by $E T(A)$-receptor antagonist. Circulation. 1997;96:1593-7.

49. Vermes I, Spooren PF, Kalsbeek-Batenburg EM, Haanen C. In addition to von Willebrand factor and urinary albumin excretion, plasma endothelin is an indicator of endothelial dysfunction in diabetes mellitus. Diabetologia. 1993;36:472-3.

50. Canani L, Zanatta C, Gerchman F, Silva M, Gross J. Endothelin-1 levels are increased in patientes with type 2 diabetes melitus and diabetic nephropathy. Diabetologia. 2005;48:A380.

51. Cardillo C, Campia U, Bryant MB, Panza JA. Increased activity of endogenous endothelin in patients with type II diabetes mellitus. Circulation. 2002;106:1783-7.

52. Hargrove GM, Dufresne J, Whiteside C, Muruve DA, Wong NC. Diabetes mellitus increases endothelin-1 gene transcription in rat kidney. Kidney Int. 2000;58:1534-45.

53. Stehouwer CD, Fischer HR, van Kuijk AW, Polak BC, Donker AJ. Endothelial dysfunction precedes development of microalbuminuria in IDDM. Diabetes. 1995;44:561-4.

54. Park JY, et al. Induction of endothelin-1 expression by glucose: an effect of protein kinase C activation. Diabetes. 2000;49:1239-48.

55. Nicaud $V$, et al. Polymorphisms of the endothelin- $A$ and - $B$ receptor genes in relation to blood pressure and myocardial infarction: the etude cas-temoins sur I'Infarctus du Myocarde (ECTIM) Study. Am J Hypertens. 1999;12:304-10. 
56. Hocher B, et al. Effects of endothelin receptor antagonists on the progression of diabetic nephropathy. Nephron. 2001;87:161-9.

57. Marther K, Mirzamohammadi B, Lteif A, Steinberg H, Baron A. ET-1A receptor blockade improves endothelium-dependent vasodilation in insulin resistant obese and type 2 diabetic patients- endothelin-1A. Diabetes. 2000;51.
Endereço para correspondência:

Luís H. Canani

Serviço de Endocrinologia do Hospital de Clínicas de Porto Alegre

Rua Ramiro Barcelos, 2.350, prédio 12, $4^{\circ}$ andar

90035-003 Porto Alegre, RS

E-mail: luiscanani@terra.com.br 